\title{
Star clusters in the dwarf irregular galaxy Leo A
}

\author{
R. Stonkutè ${ }^{1,2}$, R. Naujalis ${ }^{1}$, M. Čeponis ${ }^{1}$, A. Leščinskaité ${ }^{1}$, and V. Vansevičius ${ }^{1,2}$ \\ 1 Center for Physical Sciences and Technology, Saulètekio av. 3, 10257 Vilnius, Lithuania \\ e-mail: vladas.vansevicius@ff.vu.1t \\ 2 Vilnius University Observatory, Čiurlionio 29, 03100 Vilnius, Lithuania
}

Received 15 April 2018 / Accepted 20 May 2019

\begin{abstract}
Context. Leo A is an isolated gas-rich dwarf irregular galaxy of low stellar mass and metallicity residing at the outskirts of the Local Group. Ages of the stellar populations in Leo A range from $\sim 10 \mathrm{Myr}$ to $\sim 10 \mathrm{Gyr}$. So far, only one star cluster has been discovered in this galaxy.

Aims. Our aim is to search for star cluster candidates in the Leo A galaxy.

Methods. We performed photometry of star-like objects on HST ACS archive observation frames in the $F 475 W$ and $F 814 W$ passbands and studied the spatial clustering of the Leo A stars down to the turn-off point of the old stellar populations.

Results. We report the discovery of four star clusters in the Leo A galaxy. This means that now a sample of five star clusters is known in Leo A. Two clusters are of a young age ( $\sim 20 \mathrm{Myr}$; overlapping with $\mathrm{H}_{\text {II }}$ regions) and low in mass ( $\gtrsim 400 M_{\odot}$ and $\left.\gtrsim 150 M_{\odot}\right)$, the other three clusters are older $(\gtrsim 100 \mathrm{Myr})$ and also of low mass $\left(\gtrsim 300 M_{\odot}\right)$. These rough estimates are made assuming the isochrones of a metallicity derived for $\mathrm{H}$ II regions $(Z=0.0007)$. Colour-magnitude diagrams of the stars residing in the circular areas of a $2.5^{\prime \prime}$ radius around the clusters and integrated aperture photometry results of the clusters are presented.
\end{abstract}

Key words. galaxies: dwarf - galaxies: star clusters: general - galaxies: individual: Leo A (DDO 69)

\section{Introduction}

Studies of star clusters in low-metallicity environments help us to understand star formation processes in the high-redshift Universe. The issues of star cluster formation and evolution in various types of galaxies have been broadly discussed recently by Renaud (2018), where the importance of star cluster evolution in low-metallicity dwarf galaxies, resembling conditions of star formation in the early Universe, was stressed. One of the best targets for testing various star cluster formation scenarios at an extremely low metallicity is the nearby dwarf irregular galaxy Leo A. However, previous studies reported only one star cluster in this galaxy (Stonkute et al. 2015) that was discovered in the deep Hubble Space Telescope (HST) Advanced Camera for Surveys (ACS) images (Cole et al. 2007). In this paper we report the discovery of four new star cluster candidates that we found in the same set of HST ACS images.

Leo A (Fig. 1) is an isolated dwarf irregular galaxy in the Local Group. It is a gas-rich (Hunter et al. 2012) star system dominated by dark matter (Brown et al. 2007) with a low metallicity (van Zee et al. 2006; Kirby et al. 2017; Ruiz-Escobedo et al. 2018). The present-day low star formation activity is indicated by the few $\mathrm{H}_{\text {II }}$ regions, while the existence of an old stellar population is proven by the detection of RR Lyr stars (Dolphin et al. 2002; Bernard et al. 2013). Stellar photometry performed with the HST Wide Field and Planetary Camera 2 (WFPC2; Tolstoy et al. 1998; Schulte-Ladbeck et al. 2002) revealed an unusual star formation history $(\mathrm{SFH})$ in Leo A: the galaxy is dominated by relatively young ( $\lesssim 4 \mathrm{Gyr}$ ) stellar populations. Deep stellar photometry below the turn-off of the oldest populations performed with the HST ACS (Cole et al. 2007) confirmed previous findings and established an SFH scenario of a "young galaxy". The outer parts of the galaxy were studied with the Subaru Suprime-Cam by Vansevičius et al. (2004) and the HST Wide Field Camera 3 (WFC3) by Stonkute et al. (2018), and revealed a presence of an extended (up to $10^{\prime}$ ) elliptical stellar envelope.

The basic parameters of the Leo A galaxy, derived from the RGB star distribution (Vansevičius et al. 2004), are adopted in this study: centre coordinates of the galaxy, $\alpha=9^{\mathrm{h}} 59^{\mathrm{m}} 24^{\mathrm{s}}$, $\delta=+30^{\circ} 44^{\prime} 47^{\prime \prime}(\mathrm{J} 2000)$; an ellipticity, that is, the ratio of the semi-minor to the semi-major axis, $b / a=0.6$; and a position angle of the major axis, $\mathrm{PA}=114^{\circ}$. The distance to Leo A of $800 \mathrm{kpc}\left(1^{\prime} \approx 230 \mathrm{pc}\right.$; Dolphin et al. 2002) is based on RR Lyrae stars. The foreground Milky Way (MW) extinction estimates towards Leo A are taken from Schlafly \& Finkbeiner (2011), $A(F 475 W)=0.068$ and $A(F 814 W)=0.032$. The parameters of Leo A are summarised in Table 1.

The structure of the paper is the following: Sect. 2 presents details of the archive observation data, reductions, and stellar photometry. Section 3 presents the results of star cluster detection and the determination of their parameters. Conclusions are presented in Sect. 4.

\section{Observation data and stellar photometry}

Stellar photometry and integrated photometry of star clusters were performed using HST ACS frames in the $F 475 \mathrm{~W}$ and $F 814 W$ passbands from the project Local Cosmology from Isolated Dwarfs $\left(\mathrm{LCID}^{1}\right)$, see Cole et al. (2007) for observation details.

The archival data were downloaded from the Mikulski Archive for Space Telescopes (MAST). We retrieved

http://www.iac.es/proyecto/LCID 


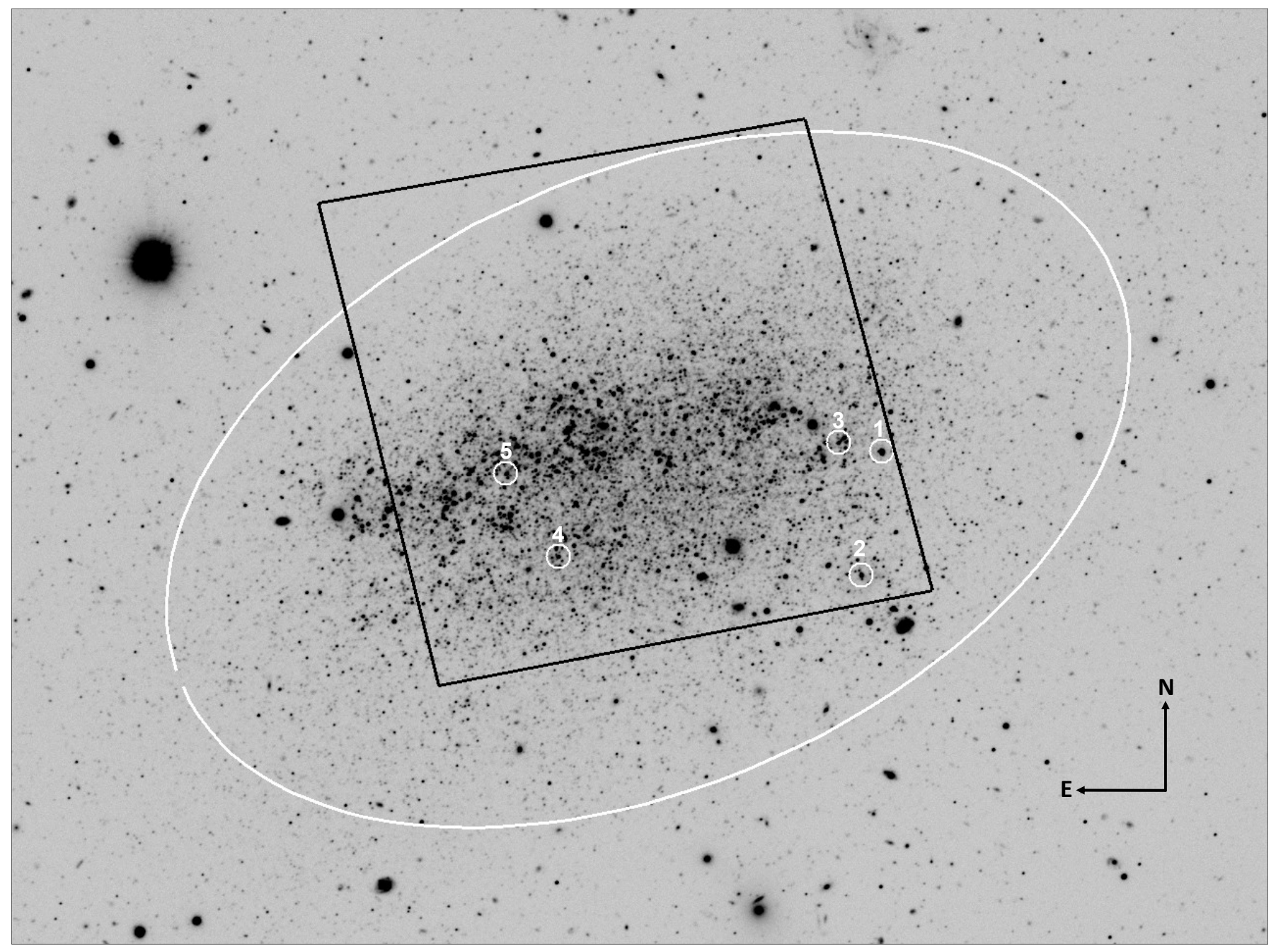

Fig. 1. Subaru Suprime-Cam $B$-passband image of the Leo A galaxy (Stonkute et al. 2014). The ellipse $\left(b / a=0.6\right.$; PA $\left.=114^{\circ}\right)$ of the semi-major axis equal to the Holmberg radius, $a=3.5^{\prime}$ (Mateo 1998), centred at $\alpha=9^{\mathrm{h}} 59^{\mathrm{m}} 24^{\mathrm{s}}, \delta=+30^{\circ} 44^{\prime} 47^{\prime \prime}(\mathrm{J} 2000)$, is shown by the white line. The HST ACS field (Cole et al. 2007) is marked by the black line. Discovered star clusters are marked by white open circles. North is up, east is left.

bias-subtracted, flat-fielded, charge transfer efficiency (CTE) corrected HST ACS flc images produced by the STScI "onthe-fly reprocessing" (OTFR) pipeline OPUS versions 2015_2b, which used CALACS version 8.3.0.

To perform stellar photometry we used the software package DOLPHOT 2.0 (Dolphin 2000, and many unpublished updates). We followed the recommended preprocessing steps and the photometry recipe provided in the manual for the HST ACS module (version of 24 February 2016). We used AstroDrizzle 1.1.16 (default parameter values) to create clean, deep-drizzled reference frames for object detection and coordinate transformations from 16 sub-exposures in each of the $F 475 W$ and $F 814 W$ passbands. This also allowed us to flag cosmic rays in the individual flc images and to update data quality images.

We used the values of the DOLPHOT parameters recommended in the HST ACS manual: the FitSky parameter was set to 1 , which means the sky fitting in an annulus around each star $\left(R_{\text {inner }}=15, R_{\text {outer }}=35\right.$ pixels $)$ and the point-spread function (PSF) fitting inside a radius of $R_{\text {apert }}=4$ pixels.

DOLPHOT determines magnitudes, magnitude errors, object fit, and shape parameters in individual flc frames, and then combines them per filter. To combine the magnitudes we set a parameter FlagMask = 5, which means that only measurements with error flags equal to 0 (excellent photometric quality) and 2 (bad or saturated pixels are present) were used.

In order to optimise the parameters for the photometry, we performed numerous tests with various source detection thresholds (SigFind and SigFinal), and a minimum allowed separation for two stars (RCombine). The best photometry quality was achieved with SigFind $=1.5$, SigFinal $=4$, and RCombine $=1.5$. These parameter values were applied for the final photometry. We also set the parameter Force1 = 1 (all detected sources are fitted as stars), as is suggested for crowded field photometry, and for the further analysis, we selected only stars with the object type flag equal to 1 and a signal-to-noise ratio $\geq 5.0$.

The initial photometry catalogue contained measurements in two passbands of 189947 objects. In order to clean the photometry catalogue, combined mosaic images were visually inspected, and objects falling on obvious background galaxies, bright MW stars, or image artefacts, as well as those residing closer to the frame borders than $2 \times R_{\text {apert }}=8$ pixels, were rejected. In order to further clean the catalogue from extended objects, we rejected objects by sharpness in both passbands $(0.2<$ sharpness $_{F 814 W}<-0.2$ and $0.2<$ sharpness $_{F 457 W}<-0.2$ ). This left us with $N=151146$ objects. 
Table 1. Parameters of the Leo A galaxy.

\begin{tabular}{lll}
\hline \hline Parameter & Value & Reference \\
\hline$\alpha(\mathrm{J} 2000) ; \delta(\mathrm{J} 2000)$ & $9: 59: 24.0 ;+30: 44: 47$ & Vansevičius et al. (2004), RGB stars \\
$b / a ; \mathrm{PA}$ & $0.60 ; 114^{\circ}$ & Vansevičius et al. (2004), RGB stars \\
$(m-M)_{0}$ & $24.51 \pm 0.12(0.80 \pm 0.04 \mathrm{Mpc})$ & Dolphin et al. (2002), RR Lyrae stars \\
$A(F 475 W) ; A(F 814 W)$ & $0.068 ; 0.032$ & Schlafly \& Finkbeiner (2011) \\
$M_{\mathrm{STARS}}$ & $3.3 \times 10^{6} M_{\odot}$ & Kirby et al. (2017) \\
$M_{\mathrm{H}}$ & $6.9 \times 10^{6} M_{\odot}$ & Hunter et al. (2012) \\
$M / L_{V}$ & $20 \pm 6 M_{\odot} / L_{\odot}$ & Brown et al. (2007), B supergiants and H II zones \\
$12+\log (\mathrm{O} / \mathrm{H})$ & $7.38 \pm 0.1$ & van Zee et al. (2006), a planetary nebula and H II zones \\
$12+\log (\mathrm{O} / \mathrm{H})$ & $7.4 \pm 0.2$ & Ruiz-Escobedo et al. (2018), H II zones \\
$<[\mathrm{Fe} / \mathrm{H}]>$ & $-1.67_{-0.08}^{+0.09}$ & Kirby et al. (2017), RGB stars \\
\hline
\end{tabular}

Notes. $\alpha(\mathrm{J} 2000)$ and $\delta(\mathrm{J} 2000)$ are the equatorial coordinates of the galaxy centre; b/a is the ratio of minor to major axes; PA is the position angle of the major axis; $(m-M)_{0}$ is the true distance modulus; $A(F 475 W)$ and $A(F 814 W)$ are the foreground extinction in corresponding passbands; $M_{\mathrm{STARS}}$ is the stellar mass; $M_{\mathrm{H}}$ is the mass of neutral hydrogen; $M / L_{V}$ is the lower limit of the mass-to-luminosity ratio; $12+\log (\mathrm{O} / \mathrm{H})$ is the oxygen abundance; and $<[\mathrm{Fe} / \mathrm{H}]>$ is the average metallicity.

\section{Results and discussions}

Compact star cluster recognition in galaxies at the outskirts of the Local Group based on images, taken even at observatories located in the best astroclimate conditions, is a very difficult task (Narbutis et al. 2008; Stonkutè et al. 2008). To search for the compact low-mass star clusters in the Leo A galaxy we therefore used available HST ACS archive images in the $F 475 \mathrm{~W}$ and $F 814 W$ passbands and colour-magnitude diagrams (CMDs) constructed from the stellar photometry data described in Sect. 2.

To search for star clusterings we selected reliably identified and measured stars $(F 814 W<28)$, and excluded the reddest stars $(F 475 W-F 814 W>2.0)$, which most probably are foreground MW stars. In total, we used 98525 stars within the HST ACS field and analysed their clustering. We used three circular cluster kernel sizes of radii $0.50^{\prime \prime}, 0.75^{\prime \prime}$, and $1.00^{\prime \prime}$ in order to reveal compact $(\$ 2 \mathrm{pc})$ and more extended $(\sim 4 \mathrm{pc})$ star clusterings. The individual background number density for each kernel position was estimated in an annulus from $1.0^{\prime \prime}$ to $2.0^{\prime \prime}$ around the kernel centre, and then was subtracted from the number density determined within the kernel. As a result, three number density maps were produced and intercorrelated. The star number density map obtained using the kernel of $0.50^{\prime \prime}$ radius contained all significant star clusterings seen in two other maps derived with larger kernels. The further analysis was therefore based on this star number density map.

As the first step, we calculated the ratio of star number densities within a radius of $0.5^{\prime \prime}$ and an annulus background zone from $1.0^{\prime \prime}$ to $2.0^{\prime \prime}$ radius for each pixel. Arbitrarily assuming a lower threshold for the star number density ratio equal to 5 , we ended up with 76 pre-selected star clusterings worth studying in more detail. However, a careful inspection of the preselected candidates on the HST ACS mosaics independently by five team members, using the by-eye procedure described in detail by Johnson et al. (2012), left us with the 5 most prominent star cluster candidates. It is worth noting, however, that the super-position of stars could only marginally affect identification reliability of cluster candidates because of well-resolved stellar populations and a comfortably oriented galaxy disk.

The colour images of the clusters and their CMDs (Fig. 2) show the star-like objects (filled black circles in CMDs) residing inside the green circle that marks the cluster itself and star-like objects (open circles) residing inside the circle of $2.5^{\prime \prime}$ radius. The colour images of clusters are constructed from the HST ACS frames taking images in the $F 814 W,(F 475 W+F 814 W) / 2$, and $F 475 W$ passbands as an input to RGB channels.

The determination of an accurate centre position of the wellresolved star cluster is a sensitive procedure. For this purpose we used peaks on the spatially smoothed star number density map at the positions of suspected star clusters. An integrated growing circular aperture (the aperture radius increases by $0.1^{\prime \prime}$ up to the radius of $2.5^{\prime \prime}$ ) photometry around these positions was performed, and radii of the first sudden flattening of the growth curves were determined for each cluster candidate. These radii were assumed as measures of cluster sizes (the parameter $R$ in Table 2). The half-light radii $R_{\mathrm{h}}$ were measured on photometric growth curves at the levels corresponding to the magnitudes derived at radii $R$ plus 0.75 . The radii of star clusters $(R$ and $R_{\mathrm{h}}$ ) estimated independently from the photometric growth curves in the $F 475 W$ and $F 814 W$ passbands coincide well within half of the applied aperture growth step, $0.05^{\prime \prime}$. We note, however, that for the cluster LeoA-C2, the growth curve based on the star number count was used instead because a peculiar photometric growth curve arose as a result of much fainter stars that surrounded an extremely bright star.

The integrated magnitudes and colour indices, derived through the circular apertures of radii $R$ and $2 \times R_{\mathrm{h}}$, are listed in Table 2. The estimated accuracy of the integrated aperture photometry (magnitudes and colour indices), taking into account the errors in background level and magnitude zero-point, is $\sim 0.05$. Moreover, the correct sky background subtraction is critical for determining the shape of the photometric growth curves in the outer regions of the clusters; for a detailed discussion, see Hill \& Zaritsky (2006). In order to estimate possible errors of the sky background subtraction, we constructed the cluster photometric profiles with sky background estimates derived in annuli of different sizes in the radii range of $1.5-3.0^{\prime \prime}$. However, this led to relatively small half-light radii changes: $\Delta R_{\mathrm{h}} \sim \pm 0.05^{\prime \prime}$.

To estimate the age of star clusters, we used PARSEC isochrones, release v1.2S (Bressan et al. 2012), with $Z=$ 0.0007 metallicity. Isochrones of $20 \mathrm{Myr}$ (a cyan line), $100 \mathrm{Myr}$ (a blue line), and $500 \mathrm{Myr}$ (a red line) are shown in all panels of Fig. 2 for reference. The isochrones are adjusted assuming the Leo A distance modulus of 24.51 (Dolphin et al. 2002) and a foreground MW extinction, $A(F 475 W)=0.068$ and $A(F 814 W)=0.032$ (Schlafly \& Finkbeiner 2011). The decision to use isochrones of $Z=0.0007$ metallicity is based on the 


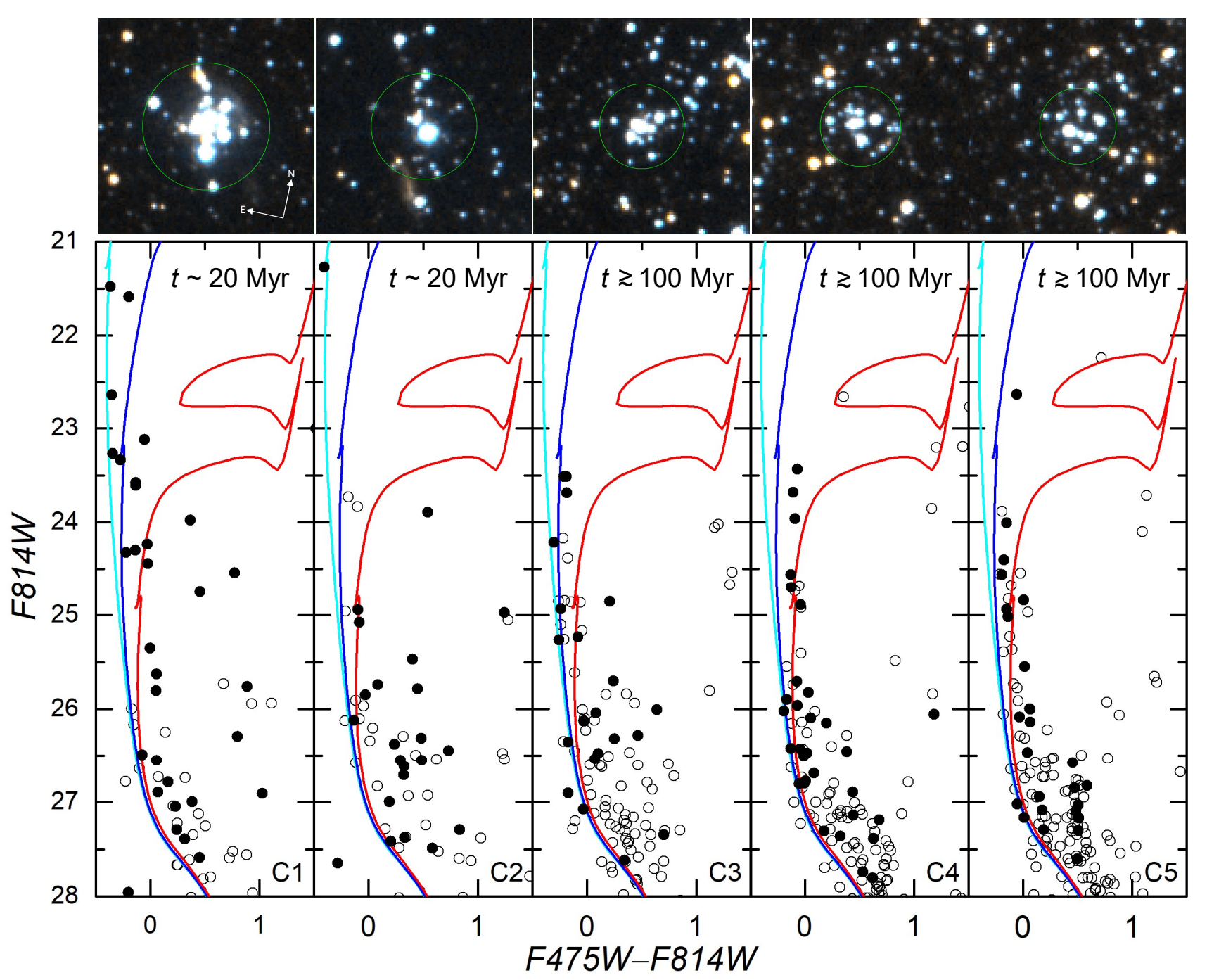

Fig. 2. Colour images $\left(5^{\prime \prime} \times 5^{\prime \prime}\right)$ of the clusters (identification codes are shown in lower right corners of each panel) and their CMDs showing the star-like objects residing inside the green circle (radius is equal to $R$, see Table 2) marking the cluster itself (filled black circles) and star-like objects residing inside the circle of $2.5^{\prime \prime}$ radius (open circles). The colour images of clusters are constructed from the HST ACS frames taking images in the $F 814 W,(F 475 W+F 814 W) / 2$, and $F 475 W$ passbands as an input to RGB channels. The PARSEC isochrones, release v1.2S (Bressan et al. 2012), of $Z=0.0007$ metallicity and ages of $20 \mathrm{Myr}$ (the cyan line), $100 \mathrm{Myr}$ (the blue line), and $500 \mathrm{Myr}$ (the red line) are shown in all panels. All isochrones are adjusted for the distance modulus of 24.51 and MW foreground reddening, $A(F 475 W)=0.068$ and $A(F 814 W)=0.032$.

oxygen abundance $(12+\log (\mathrm{O} / \mathrm{H})=7.4)$ determined for $\mathrm{H}_{\mathrm{II}}$ regions in Leo A by van Zee et al. (2006) and Ruiz-Escobedo et al. (2018). We note, however, that the young ages estimated from the upper main-sequence stars of the clusters are rather insensitive to the assumed metallicity. An independent strong support of the young ages of the LeoA-C1 and LeoA-C2 clusters is their location in the areas of prominent $\mathrm{H}_{\text {II }}$ zones (Fig. 3).

Based on the ages of the star clusters, we estimated their approximate mass to be in the range of $\sim 200-500 M_{\odot}$ (Table 2). The mass of star clusters was determined from the distribution of the blue $(F 475 W-F 814 W<0.5)$ bright $(F 814 W<26)$ stars located on or near the main sequence (Fig. 2). We applied the initial mass function by Kroupa (2002) in the stellar mass range from 0.08 to $120 M_{\odot}$.

In order to estimate the fraction of stars that form in clusters, we employed the recent SFH in Leo A derived within the area of the HST ACS field (Čeponis et al. 2018). Taking into account two young clusters (LeoA-C1 and LeoA-C2) and stars formed during the last $30 \mathrm{Myr}$, we determine that $\sim 10 \%$ of stars were formed in clusters. Taking into account all five clusters (LeoA$\mathrm{C} 1-\mathrm{C} 5$ ) and stars formed during the last $200 \mathrm{Myr}$, we derive that $\sim 2 \%$ of stars were formed in clusters. A large difference of these estimates arises, most probably, from selection effects (detection incompleteness of older clusters) and cluster destruction or dissolution processes.

Finally, we would like to stress that all physical parameters derived for the low-mass $\left(\sim 300 M_{\odot}\right)$ star clusters are subject to strong stochastic effects (de Meulenaer et al. 2014) and should be treated carefully just as best-guess estimates.

\section{Conclusions}

The aim of this study was to search for compact star cluster candidates in the dwarf irregular galaxy Leo A, which has an extremely low metallicity (Kirby et al. 2017). So far, only one star cluster (LeoA-C1), discovered recently by Stonkute et al. (2015), was known in this galaxy.

We have performed photometry of star-like objects on HST ACS archive frames in the $F 475 W$ and $F 814 W$ passbands 
Table 2. Parameters of star clusters in Leo A.

\begin{tabular}{|c|c|c|c|c|c|c|c|c|c|c|}
\hline $\begin{array}{l}\text { ID } \\
(1)\end{array}$ & $\begin{array}{c}\alpha(\mathrm{J} 2000) \\
(2)\end{array}$ & $\begin{array}{c}\delta(\mathrm{J} 2000) \\
(3)\end{array}$ & $\begin{array}{c}R\left[{ }^{\prime \prime}\right] \\
(4)\end{array}$ & $\begin{array}{c}R_{\mathrm{h}}\left[{ }^{\prime \prime}\right] \\
(5) \\
\end{array}$ & $\begin{array}{c}F 814 W \\
(6)\end{array}$ & $\begin{array}{c}F 814 W 2 R_{\mathrm{h}} \\
(7)\end{array}$ & $\begin{array}{l}C I \\
(8) \\
\end{array}$ & $\begin{array}{c}C I_{2 R_{\mathrm{h}}} \\
(9)\end{array}$ & $\begin{array}{c}\text { Age (Myr) } \\
(10)\end{array}$ & $\begin{array}{c}\text { Mass }\left(M_{\odot}\right) \\
\quad(11)\end{array}$ \\
\hline LeoA-C1 & $9: 16.5$ & $+30: 44: 59$ & 1.50 & 0.55 & 19.85 & 19.96 & -0.11 & -0.11 & $\sim 20$ & $z 400$ \\
\hline LeoA-C2 & $9: 59: 17.2$ & $+30: 44: 08$ & 1.25 & $0.7^{*}$ & 20.82 & 20.79 & -0.24 & -0.23 & $\sim 20$ & $\gtrsim 150$ \\
\hline LeoA-C3 & $9: 59: 17.9$ & $+30: 45: 02$ & 1.00 & 0.31 & 21.69 & 21.86 & -0.15 & -0.14 & $\gtrsim 100$ & $\gtrsim 300$ \\
\hline LeoA-C4 & $9: 59: 26.9$ & $+30: 44: 15$ & 0.95 & 0.37 & 21.68 & 21.89 & 0.02 & -0.06 & $\gtrsim 100$ & $\approx 300$ \\
\hline LeoA-C5 & $9: 59: 28.5$ & $+30: 44: 50$ & 0.90 & 0.34 & 21.78 & 21.85 & -0.14 & -0.15 & $\gtrsim 100$ & $\geq 300$ \\
\hline
\end{tabular}

Notes. (1) ID is the cluster identification number; (2) $\alpha(\mathrm{J} 2000)$ and (3) $\delta(\mathrm{J} 2000)$ are the equatorial coordinates; (4) $R$ is the radius of the cluster in arcsec (in Fig. 2 it is marked with a green circle); (5) $R_{\mathrm{h}}$ is the half-light radius of the cluster derived from the photometric growth curve in $\operatorname{arcsec}$ (the asterisk shows the half-light radius of the cluster LeoA-C2 estimated from the star number count); (6) $F 814 \mathrm{~W}$ is the magnitude measured through the aperture of radius $R$; (7) $F 814 W_{2 R_{\mathrm{h}}}$ is the magnitude measured through the aperture of radius $2 \times R_{\mathrm{h}}$; (8) $C I$ is the colour index $F 475 \mathrm{~W}-F 814 \mathrm{~W}$ measured through the aperture of radius $R$; (9) $C I_{2 R_{\mathrm{h}}}$ is the colour index $F 475 \mathrm{~W}-F 814 \mathrm{~W}$ measured through the aperture of radius $2 \times R_{\mathrm{h}} ;(10)$ age (Myr) is a rough estimate of the cluster age in Myr; and (11) mass $\left(M_{\odot}\right)$ is a rough estimate of the cluster mass in solar masses, $M_{\odot}$.
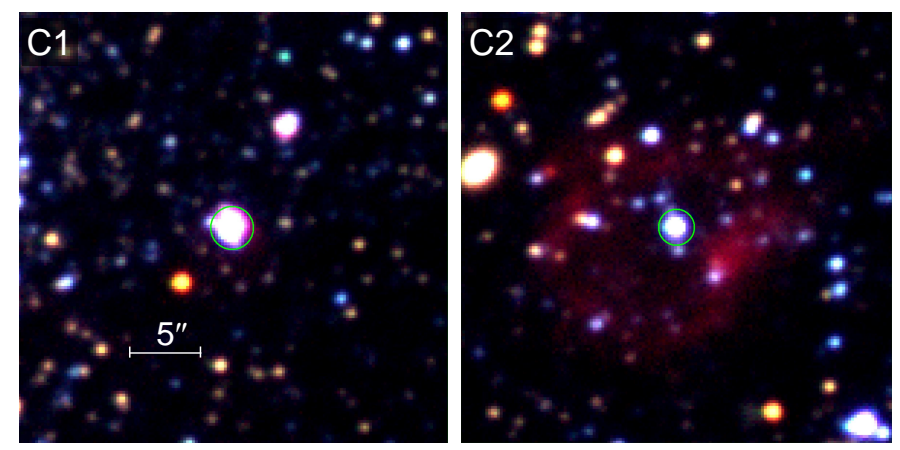

Fig. 3. Composite colour images $\left(30^{\prime \prime} \times 30^{\prime \prime}\right)$ of star clusters LeoA-C1 and LeoA-C2. The colour images of the clusters are made from the Subaru Suprime-Cam frames (Stonkute et al. 2014) taken in the $H \alpha, V$, and $B$ passbands as input to the RGB channels. The green circles that mark the clusters are of the same size as in Fig. 2. North is up, east is left.

covering the centre of Leo A and have studied the spatial clustering of the stars down to the turn-off point of old stellar populations. We found four new compact low-mass star cluster candidates. Based on the cluster CMDs analysis applying the isochrones (Bressan et al. 2012) with metallicity $Z=$ 0.0007, which is derived for $\mathrm{H}_{\text {II }}$ regions (van Zee et al. 2006; Ruiz-Escobedo et al. 2018), relatively young cluster ages and low masses were estimated (LeoA-C2: $\sim 20 \mathrm{Myr}$ and $\gtrsim 150 M_{\odot}$; LeoA-C3-C5: $\gtrsim 100 \mathrm{Myr}$ and $\left.\gtrsim 300 M_{\odot}\right)$.

The finding of such low-mass $\left(\sim 300 M_{\odot}\right)$ and young- to intermediate-age ( 20-100 Myr) star clusters in the low stellar mass $\left(3.3 \times 10^{6} M_{\odot}\right)$ and the extremely low metallicity $(12+$ $\log (\mathrm{O} / \mathrm{H})=7.4)$ dwarf irregular galaxy Leo A (in which $\mathrm{CO}$ emission has not been detected so far) could help constrain star formation scenarios in the early Universe. The problems of lowmass star clusters that reside in similar environments have been addressed only recently: an extensive study of star clusters in the LEGUS dwarf galaxies has been published by Cook et al. (2019). The Leo A galaxy is at the lowest mass and at the lowest metallicity limits of the LEGUS dwarf galaxies. Therefore, clusters discovered in Leo A consistently extend the parameter space of star clusters measured in the LEGUS dwarfs.

However, in order to determine a complete census of star clusters in the Leo A galaxy, a much larger field (about four times larger), observed with the resolution of the HST ACS or
WFC3 cameras, is needed. This conclusion is supported by the number of additional star cluster candidates seen in the Subaru Suprime-Cam frames, for instance, the obvious young star cluster embedded in the $\mathrm{H}$ II region resides just outside the HST ACS field; see $\sim 8^{\prime \prime}$ to the north-west of LeoA-C1 in Fig. 3.

Acknowledgements. We thank the anonymous referee for helpful suggestions that improved the presentation of this paper. The research has made use of the SAOImage DS9, developed by Smithsonian Astrophysical Observatory. The data presented in this paper were obtained from the Multimission Archive at the Space Telescope Science Institute. This research was funded by a grant (No. LAT-09/2016) from the Research Council of Lithuania.

\section{References}

Bernard, E. J., Monelli, M., Gallart, C., et al. 2013, MNRAS, 432, 3047 Bressan, A., Marigo, P., Girardi, L., et al. 2012, MNRAS, 427, 127 Brown, W. R., Geller, M. J., Kenyon, S. J., \& Kurtz, M. J. 2007, ApJ, 666, 231 Čeponis, M., Stonkutè, R., \& Vansevičius, V. 2018, in Dwarf Galaxies: from the Deep Universe to the Present, eds. S. Stierwalt, \& K. McQuinn, Proc. IAU Symp., 344

Cole, A. A., Skillman, E. D., Tolstoy, E., et al. 2007, ApJ, 659, L17 Cook, D. O., Lee, J. C., Adamo, A., et al. 2019, MNRAS, 484, 4897 de Meulenaer, P., Narbutis, D., Mineikis, T., \& Vansevičius, V. 2014, A\&A, 569, A4

Dolphin, A. E. 2000, PASP, 112, 1383

Dolphin, A. E., Saha, A., Claver, J., et al. 2002, AJ, 123, 3154

Hill, A., \& Zaritsky, D. 2006, AJ, 131, 414

Hunter, D. A., Ficut-Vicas, D., Ashley, T., et al. 2012, AJ, 144, 134

Johnson, L. C., Seth, A. C., Dalcanton, J. J., et al. 2012, ApJ, 752, 95

Kirby, E. N., Rizzi, L., Held, E. V., et al. 2017, ApJ, 834, 9

Kroupa, P. 2002, Science, 295, 82

Mateo, M. L. 1998, ARA\&A, 36, 435

Narbutis, D., Vansevičius, V., Kodaira, K., Bridžius, A., \& Stonkutè, R. 2008, ApJS, 177, 174

Renaud, F. 2018, New A Rev., 81, 1

Ruiz-Escobedo, F., Peña, M., Hernández-Martínez, L., \& García-Rojas, J. 2018, MNRAS, 481, 396

Schlafly, E. F., \& Finkbeiner, D. P. 2011, ApJ, 737, 103

Schulte-Ladbeck, R. E., Hopp, U., Drozdovsky, I. O., Greggio, L., \& Crone, M. M. 2002, AJ, 124, 896

Stonkutè, R., Vansevičius, V., Arimoto, N., et al. 2008, AJ, 135, 1482

Stonkutè, R., Arimoto, N., Hasegawa, T., et al. 2014, ApJS, 214, 19

Stonkutè, R., Narbutis, D., Bridžius, A., Leščinskaitè, A., \& Vansevičius, V. 2015, Baltic Astron., 24, 293

Stonkutè, R., Čeponis, M., Leščinskaitė, A., Naujalis, R., \& Vansevičius, V. 2018, A\&A, 614, A144

Tolstoy, E., Gallagher, J. S., Cole, A. A., et al. 1998, AJ, 116, 1244

Vansevičius, V., Arimoto, N., Hasegawa, T., et al. 2004, ApJ, 611, L93

van Zee, L., Skillman, E. D., \& Haynes, M. P. 2006, ApJ, 637, 269 